Aus der oto-laryngologisehen Klinik des Communehospitals zu Kopenhagen (Director: Dr. Holger Mygind).

\title{
Ein Fall von labyrinthärer Angioneurose, von Auftreten des Menière'schen Symptomencomplexes begleitet.
}

\author{
Von \\ Jörgen Moller, \\ Assistenzarzt der EliniL.
}

Folgender, an der oto-laryngologischen Klinik des Kopenhagener Communehospitals wahrgenommener Fall bietet in mehreren Richtungen ein so grosses Interesse dar, dass er es verdient veröffentlicht zu werden.

Die Krankengeschichte ist in kurzen Zügen folgende:

N. N., 42jähriger Lebrer, ist fruhher im Wesentlichen gesund gewesen and hat namentlich viemals Zeichen irgend eines Ohrleidens bemerkt. 1886 hatte er ein Dleus induratum penis, und 1 Monat später fleckiges Exanthem und wurde deshalb mit 60 Quecksilbereinreibungen behandelt. 1887 und 1888 abermalige Hautefflorescenzen. 1888 fand sich eine vorübergehende Lähmung des einen Beines ein. Der Kranke hat später geheirathet und gesunde Kinder gezeugt. 1898 ist er wegen einer Hypertrophie der Schleimhaut der beiden Conchae inferiores behandelt worden. Uebrigens ist er von nervösem Temperamente, ein wenig Hypochonder and zu Congestionen zum Kopfe geneigt. Juli 1899, wo eine grosse Hitze herrachte und der Patient viel Arbeit hatte, stelite sich, nachdem er schon längere Zeit hindurch an leichter beiderseitiger Sehwerbörigkeit gelitten hatte, ohne vorhergehende Erkrankung plötalich ein starker Anfall von Schwindel ein, begleitet von starken subjectiven Gehörsempfindungen. Die Anfälle wiederholten sich 3 bis 4 Mal, mit Zwischenräumen von wenigen Tagen. Seit dem ersten Anfalle ist fortwährend continuirliches Sausen im linken Ohre vorhanden gewesen (etwa wie das Sausen des Windes). Jeder neue Schwindelanfall wird gewöhnlich von stärkeren, ebenfalls linksseitigen, subjectiven Gehörsemptindungen anderer Art (wie das Brummen einer Wespe) eingeleitet, so dass ihm Zeit genug gegönnt ist, bevor noch der Anfall eintritt, etwas zu finden, woran er sich stiutzen könne. Die Anfalle dauern meist nur kürzere Zeit, einige Minuten, sind nicht von Bewusstlosigkeit, dagegen öfters von, sogar einmal eine halbe Stunde anhaltendem Erbrechen begleitet. Der Patient hat keine Neigung gespürt, nach einer bestimmten Seite hin zu fallen. Ob während der Anfälle stärkere Schwerhörigkeit als sonst vorbanden sei, lässt sich nicht entscheiden, da der Patient von dem Anfalle selbst zu stark in Anspruch genommen ist, als dass er hieruber Wahrnehmungen machen kann. Die Schwindelanfälle fanden sich fortwährend mit verschieden langen $Z$ wischenräumen ein, wurden aber im November entschieden häufiger. Die Schwerhörigkeit hat unverändert fortgedanert. Von den beschriebenen Erscheinungen abgesehen, haben sich nur zuweilen leichtere Störungen der Verdauungsorgane (besonders Stuhlverstopfung) eingefunden, ubrigens hat er sich völlig wohl gefühlt. 
In der letzten Hälfte des Novembermonats kam der Patient in die Klinik zur Behandlung. Bei der Untersuchung wurde gefunden:

A uris dextra: Hammergriff natürlich gestellt; der vordere Theil des Trommelfelles weiss, verdickt, beinahe glanzlos, ohne Lichtkegel; der hintere Theil atrophisch, durchscheinend, von dem vorderen Theile mittelst einer bogenförmigen Linie scharf abgegrenzt.

A uris sinistra: Hammergriff etwas ein- und aufwärts gezogen. Trommelfell ein wenig verdickt und matt, Lichtkegel mit vermindertem Glanze, körnig; dem Hammergriff entlang ein Injectionsstreifen.

Pharynx: Recht bedeutende subacute Röthe und diffuse Schwellung.

Rhinophary $\mathrm{nx}$ : Leichter hypertrophischer Katarrh.

Cavam nasi: Einige Hypertrophie der Schleimhaut der hinteren Enden beider Conchae inferiores.

Functionsuntersuchung siehe unten.

Diagnose: Otitis media catarrhalis chronica duplex. Angioneurosis auris internae sinistrae. Pharyngitis chronica. Rhino-pharyngitis chronica. Rhinitis chronica bypertrophica.

Es wurde verordnet: Bettlage, täglich subcutane Einspritzungen von Pilocarpinum hydrochloricum 0,004 , steigend; ferner Kalium bromatum $10: 200$, täglich drei Esslöffel und Sal Carlsbadense, ein Theelöffel Morgens.

Während den ersten 5 Tagen fanden sich wiederholte Schwindelanfälle ein, immer Morgens um 4-5 Uhr; einmal sogar während einer Stunde 9 bis 10 Anfälle mit deutlichen freien $Z$ wischenräumen; später fanden sich zwar bisweilen stärkere subjective Gehörsempfindungen der früher besprochenen Art ("Brummen einer Wespe"), einmal auch eine Viertelstunde lang hohe klingende Töne, aber kein Schwindel; später verloren sich diese Erscheinungen allmählich, sowie auch das continuirliche Sausen bedeutend abnahm. Nach 20 Tagen konnte der Patient etwas ausser dem Bette seỉn, und schon am nächsten Tage ging er ein wenig aus. Ein paar Tage später wurde mit den Einspritzungen aufgehört, nachdem er 19 derselben bekommen hatte, davon 13 von Pilocarpinum hydrochloricum 0,01 , wobei ausgesprochene Wirkung mit profuser Schweissabsonderung und Salivation eingetreten. Später wurde er mit Luftdouche per tubam behandelt, und gegen Ende Februar befand er sich röllig wohl, gab an mit beiden Obren gut zu hören und hatte von subjectiven Gehörsempfindungen nur zuweilen leichtes Sausen.

Nachstehend werden beigefügt einige Ergebnisse der functionellen Untersuchung, Die bei den fünf ersten Untersuchungen angefuhrten Ferceptionszeiten beziehen sich anf eine gewöhnliche Stimmgabel $a^{\prime}$, während die letzte Untersuch ıng mit den Bezold'schen Stimmgabeln vorgenommen wurde.

27. Nov. 21. Dec. 10.Jan. 23.Jan. 30.Jan. 5. März

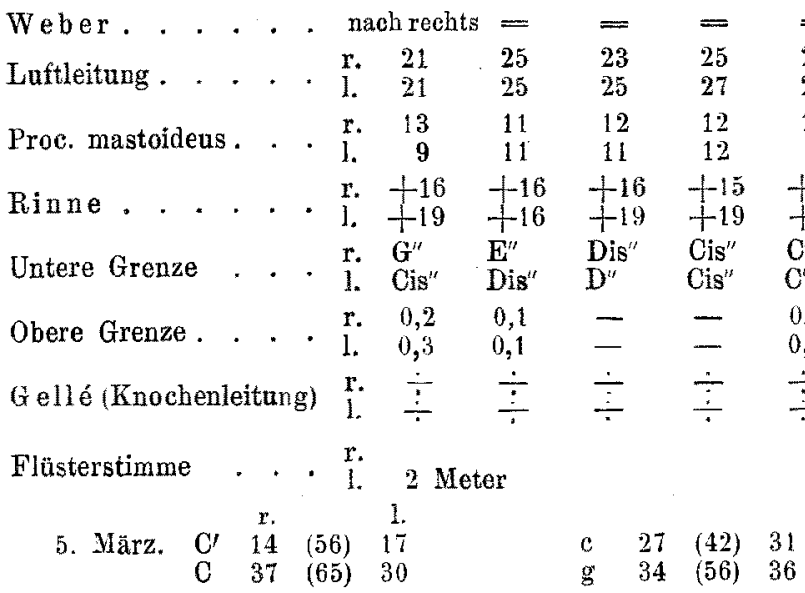




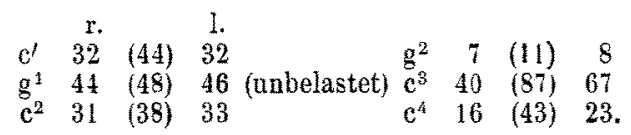

Die letate Gabel war eine gewöhnliche $c^{4}$-Gabel (nicht Bezold's).

Die in Klammern beigefügten Zahlen beziehen sich auf die Zeit, in welcher der Verfasser (welcher normalbörig ist) den betreffenden. T'on hört.

Wie man sieht, ist dieser Fall dem von Gustav Brunner') veröffentlichten und als vasomotorische Neurose der Labyrinthgefässe bezeichneten sehr ähnlich. Der Fall Brunner's war in kurzen Zügen folgender:

Ein junger Mann leidet an einem bisher unbeachteten chronischen Mittelohrkatarrhe. Plötzlich finden sich Schwindelanfälle ein, mit Zwischenräumen von einer bis mehreren Wochen auftretend. $\mathrm{Zu}$ gleicher Zeit erweist die Untersuchung ein Herabsetzen des Hörvermögens, namentlich für hohe Töne, indem sogar von der Mitte der dreigestrichenen Oetave nach aufwärts völliger Mangel jeder Tonperception besteht. Die Anfälle werden von einer Aura eingeleitet. Ansser den Schwindelanfällen treten während des spăteren Krankheitsverlaufes Anfälle von Hemianaesthesia acustica auf, meistens durch emotionelle Einwirkungen herbeigeführt. Es gelingt durch Eingabe von Chinin während der Aura die Anfälle zu coupiren. Brunner ist der Meinung, dass es sich um angioneurotische Störungen handle; doch ist es wohl zulässig, auch dem anwesenden Mittelohrenkatarrhe einige Bedeutang zuzuschreiben.2)

In unserem Falle findet sich auch ein bisher nur wenig beachteter chronischer Katarrh, weloher wohl am ehesten als eine Sklerose anzusehen ist; hierauf deutet insbesondere das Herabsetzen des Hörvermögens für hohe Töne sowie der Ausfall des Gelléschen Versuches. Das Herabsetzen des Hörvermögens ist kein so bedeutendes gewesen, dass es sich unter gewöhnliehen Verhältnissen geltend gemacht hätte, und subjective Gehörsempfindungen sind bisher nicht vorhanden gewesen. Plötzlich tritt aber, zum Theil durch äussere Verhältnisse herbeigeführt, ein Schwindelanfall auf. Versucht man es, den näheren Vorgang auseinander zu setzen, ergiebt sich Folgendes: Der Patient ist ein kräftiges, vollblütiges Individuum, von etwas nervösem Temperamente und geneigt zu vasomotorischen Störungen. Wegen starker Anstrengung bei grosser Hitze geräth sein Nervensystem in weitere Unordnung und unter anderen finden sich stärkere vasomotorische Störungen mit acuter Hyperämie des inneren Ohres ein. Hätte es sich nun nm normale Ohren gehandelt, wäre wabrscheinlich nichts Weiteres geschehen, ausser dass sich etwas Ohrensausen hätte einfinden können; jetzt aber besteht sohon im Voraus eine Sklerose, welohe das Ohr ausser Stande setzt, die

1) Zum Morbus Menière. Zeitschr. f. Ohrenheilk. XVII. 1887. S. 47.

2) Brunner erwähnt einen Fall von "angioneurotischer Acusticuslähmung", welchen Politzer gesehen hat; da derselbe aber sowohl von dem Brunner'schen ais von dem unserigen in mehreren Richtungen verschieden ist, werde ich auf ihn nicht näher eingehen. 
durch die Hyperämie herbeigeführte Druckveränderung in normaler Weise auszugleichen; es tritt dann von Seiten des Ohres eine Reaction in der Form eines "Menière'schen Anfalles" ein. Wie in dem Brunner'schen Falle findet man auch hier eine Aura, in subjectiven Gehörsempfindungen eigenthümlicher Art bestehend. Fiir das Vorhandensein einer Angioneurose spricht auch, dass die Anfälle zuweilen in bedeutender Anzahl mit nur kurzen, doch aber deutlich ausgesprochenen freien Zwischenräumen auftreten, einmal sogar bis neunmal während 1 Stunde. Ein so schnelles Wechseln des Zustandes wird man wohl schwerlich anders erklären können, als dass es eine Folge acuter Hyperämie rein vasomotorischen Ursprungs sei.

Ausser den recidivirenden Anfällen von Hyperämie des inneren Ohres muss inzwischen angenommen werden, dass anch einige stationäre, durch den chronischen Mittelohrkatarrh herbeigeführte Hyperämie der lautleitenden Abschnitte des Ohres bestehe, wodurch das continuirliche tiefere Sausen und einige Verminderung des ganzen Hörvermögens sowohl für Flüsterstimme als für Töne herbeigeführ't werden.

Bemerkenswerth ist es, dass die untere Grenze der Tonperception fur das rechte, scheinbar weniger angegriffene $\mathrm{Ohr}$ recht bedeutend emporgerückt ist, während für das linke Ohr nur ein unbeträchtliches Emporrücken besteht. Vielleicht könnte man diese Erscheinung in solcher Weise erklären, dass die Hyperämie am rechten Ohre im Wesentlichen in den lautleitenden Abschnitten, am linken dagegen mehr in dem Labyrinthe seinen Sitz habe; hiermit stimmt auch der Umstand überein, dass man bei dem Weber'schen Versuche Lateralisirung nach rechts findet.

Die Pilocarpinbehandlung hat sich in diesem Falle, wie in vielen anderen Fällen von labyrinthären Affectionen, als ein gutes Mittel bewährt, und das Endresultat war befriedigend. Die Schwindelanfälle haben aufgehört (noch Mitte April war der Kranke ohne Anfälle), so dass der Kranke seine frïhere Beschäftigung hat aufnehmen können; die subjectiven Gehörsempfindungen haben sich beinahe vollständig verloren; zurück blieben nur noch die functionellen Zeichen der anwesenden Sklerose: Die Tonperception war für die tieferen Töne vermindert, sowie auch etwas für die höchsten; für die mittleren Töne war sie annähernd normal, jedoch auch hier ein wenig vermindert; ausserdem war das Hörvermögen für die Flüsterstimme etwas herabgesetzt, und der Gellésche Versuch gab fortwährend negatives Resultat. 